CIENCIA Y SOCIEDAD

Volumen IX, Número 2

Mayo - Agos to 1984

ROTURA DE PRESAS

JULIO SANTOS-CAYADO

La construcción de presas posibilita la utilización eficiente de las aguas de un país. A medida que en un sistema fluvial se cons truyen más presas, aumenta el porcentaje de aprovechamiento del volü men anual de sus aguas, pero también aumenta el nivel técnico requerido para operar el sistema eficientemente y con seguridad.

Errores de operación, sencillos e inconsecuentes, como abrir o cerrar una compuerta a destiempo en una toma directa de un canal, por ejemplo, pueden tener repercusiones catastróficas si ésa es parte de una presa o de un sistema de presas.

Este artículo pretende llevar a la atención de las autoridades hidráulicas nacionales, así como de la Defensa Civil, el concepto de que no es suficiente diseñar, operar y mantener las presas, sino, que además, es necesario saber lo que pasaría, si esas estructuras falla ran, a fin de preparar planes de contingencia.

El conocimiento de los efectos de la rotura de una presa debe enfocarse de una manera adulta, sin alarmismos.

\title{
Estadísticas
}

Las presas, desafortunadamente, están sujetas a incidentes. 


\section{Tipos de incidentes: 1}

- Fallos, de tipos F1 y F2.

- Accidentes, tipos A1, A2 y A3.

- Accidentes-Reservoir, AR.

- Daño durante la construcción, DDC.

- Reparación mayor, MR.

F1 - Rotura mayor que implica abandono de la presa.

F2 - Rutura severa que puede ser reparada.

A1 - Accidente que no se convirtió en rotura por reparación u operación a tiempo.

A2 - Accidente observado durante el llenado inicial de la pre sa.

A3 - Accidente ocurrido antes del llenado del embalse, lago o pantano.

AR - Accidente o problemas inusuales que ocurren en el área del embalse durante la operación de la presa.

DDC - Daños a una presa o a una de sus estructuras temporales.

MR - Reparación mayor, extensiva, requerida por el deterioro - para actualizar alguna de las partes de una presa.

En los Estados Unidos de América, por ejemplo, existen 4,974 grandes presas; es decir, con una altura mayor de 15 metros; en ésas se han reportado hasta 1973 los siguientes incidentes:

Número de los tipos de incidentes por décadas ${ }^{2}$

\begin{tabular}{|c|c|c|c|c|c|c|c|c|c|}
\hline Incidente & F1 & F2 & A1 & $\mathrm{A} 2$ & A3 & AR & MR & DDC & Total \\
\hline Antes 1899 & 5 & 2 & 3 & 1 & 0 & 0 & 0 & 0 & 11 \\
\hline $1900-1909$ & 5 & 4 & 2 & 1 & 0 & 0 & 0 & 1 & 13 \\
\hline $1910-1919$ & 7 & 11 & 12 & 2 & 1 & 1 & 5 & 2 & 41 \\
\hline $1920-1929$ & 9 & 3 & 14 & 4 & 0 & 0 & 7 & 6 & 43 \\
\hline $1930-1939$ & 3 & 4 & 8 & 5 & 0 & 1 & 4 & 6 & 31 \\
\hline $1940-1949$ & 2 & 1 & 10 & 4 & 0 & 0 & 7 & 0 & 24 \\
\hline $1950-1959$ & 3 & 4 & 10 & 4 & 0 & 1 & 8 & 4 & 34 \\
\hline $1960-1965$ & 3 & 3 & 17 & 14 & 2 & 1 & 10 & 10 & 60 \\
\hline 1966-1969 & 1 & 1 & 17 & 2 & 0 & 1 & 22 & 3 & 47 \\
\hline $1970-1973$ & 1 & 2 & 11 & 8 & 1 & 0 & 18 & 4 & 45 \\
\hline TOTALES & 39 & 35 & 104 & 45 & 4 & 5 & 81 & 36 & 349 \\
\hline
\end{tabular}

1USCOLD/ASCE. Lessons from Dam Incidents, USA. New York: ASCE, 1975. 2Ibidem. 
Es decir, el $7.02 \%$ de las grandes presas existentes en los Esta dos Unidos han reportado incidentes, y el $1.49 \%$ ha fallado.

Los tipos de presas construídas y registradas en los Estados Uni dos de América hasta 1973, son los siguientes:

Tipos de presas ${ }^{3}$

Década

E $\quad$ ER $R$

G A

RAI STL UNK TOTAL

$-1899$

81

$-1909$

89

$\begin{array}{lll}9 & 5 & 22\end{array}$

22
48

5

9

$-1919$

145

194

90

54

- 1929

240

17

88

83

- 1939

228

4
13

52

53

- 1949

708

7

45

20

- 1959

985

23

89

19

- 1965

707

- 1969

36

32

19

$25 \quad 22$

227

15

21

10

2

$\begin{array}{llllll}\text { TOTALES } & 3604 & 151 & 141 & 487 & 276\end{array}$

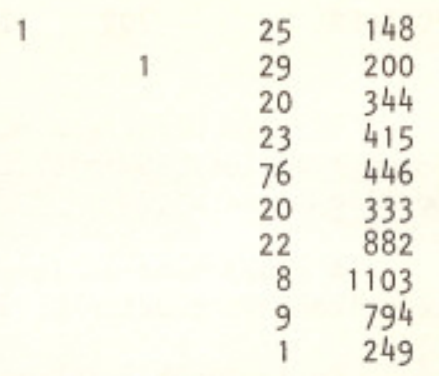

$253 \quad 4918$

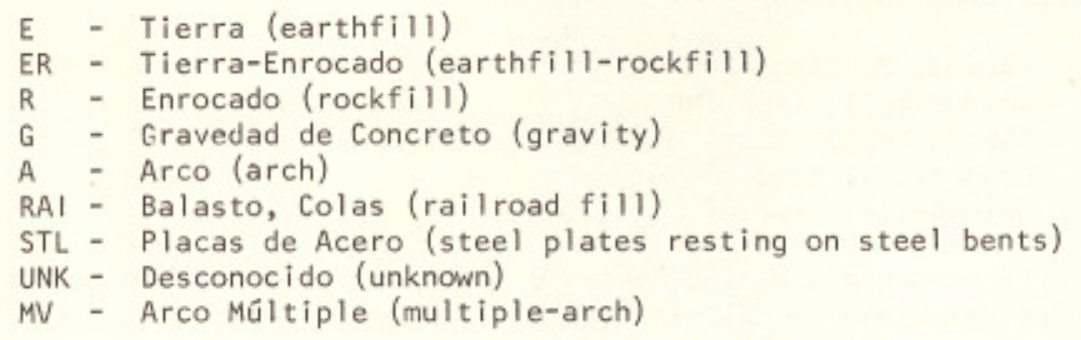

Los incidentes en los tipos de presas se distribuyen de la siguiente forma:

${ }^{3}$ Ibidem. 
Tipo de incidente para tipo de presa ${ }^{4}$

$\begin{array}{lrrrrrrr}\text { Incidente } & \text { E } & \text { ER } & \text { R } & \text { G } & \text { MV } & \text { MISC. } & \text { TOTAL } \\ \text { F1 } & 21 & 4 & 4 & 4 & 2 & 4 & 39 \\ \text { F2 } & 21 & 2 & 0 & 8 & 4 & 0 & 35 \\ \text { A1 } & 80 & 5 & 6 & 7 & 6 & 0 & 104 \\ \text { A2 } & 36 & 3 & 1 & 2 & 2 & 1 & 45 \\ \text { A3 } & 4 & 0 & 0 & 0 & 0 & 1 & 5 \\ \text { AR } & 2 & 1 & 0 & 2 & 0 & 0 & 5 \\ \text { MR } & 25 & 4 & 3 & 31 & 18 & 0 & 81 \\ \text { DDC } & 20 & 5 & 1 & 7 & 3 & 0 & 35 \\ \text { TOTALES } & 209 & 24 & 15 & 61 & 35 & 5 & 349\end{array}$

Se hace notar que no se han producido fallos de grandes presas por terremotos. Sólo se encuentran en los registros dos accidentes ti po A1 por esa causa.

A nivel mundial tampoco se han reportado fallos por movimientos telúricos; se cuentan 6,5 tipo A1 y 1 tipo A3.

En la República Dominicana tenemos 9 grandes presas: Tavera, Bao, Sabana Yegua, Valdesia, Sabaneta, Hatillo, Rincón, Majuaca y Chacuey.

Aunque en el país los datos sobre proyectos hidráulicos se guar dan celosamente, por alguna razón incomprensible, la prensa naciona ha reportado los siguientes incidentes:

Tavera, 2, Tipo A2 y MR

Valdesia, 1, tipo MR

Sabana Yegua, 1, tipo A2

Chacuey, 1, tipo A2

Majuaca, 1, tipo A2

El porcentaje de incidentes dominicanos es de $66.67 \%$, cifra que es considerablemente más al ta que el $7.02 \%$ ya citado.

Afortunadamente, en la República Dominicana no han ocurrido fa11 as, roturas, aunque durante el paso del huracán David y de la tormenta Federico, la presa de Tavera estuvo a punto.

${ }^{4}$ Ibidem. 


\section{Ecuaciones}

Para enmarcarel problema de rotura de presa dentro de las ecuaciones fundamentales de la mecánica de fluidos, recordemos la de $\mathrm{Na}-$ vier-Stokes: 5

$$
\frac{D V}{D t}=-\nabla p+\nabla^{2} \mu \bar{V}+\frac{1}{3} \nabla \mu \theta+\rho \bar{f}
$$

Para viscosidad constante, $\mu=K$ :

$\rho \frac{D \bar{V}}{D t}=-\nabla p+\mu \nabla^{2} \bar{V}+\frac{\mu}{3} \nabla \theta+\bar{f}$

Donde $\nabla^{2} \bar{v}=\nabla \theta-\nabla \times(\nabla \times \bar{v})$

Para un flujo incompresible la densidad es constante:

$$
\begin{gathered}
\theta=\operatorname{div} \bar{V}=0 \\
\frac{D \bar{V}}{D t}=-\frac{1}{\rho} \nabla p+v \nabla^{2} \bar{V}+\bar{f} \\
U=\frac{\mu}{\rho}, \text { viscosidad cinemática }
\end{gathered}
$$

La ecuación del flujo no viscoso, incompresible y no permanente en una dirección, resulta:

$$
\frac{\partial u}{\partial t}+u \frac{\partial u}{\partial x}=-\frac{1}{\rho}-\frac{\partial p}{\partial x}
$$

Donde,

$$
u=u(x, t) ; p=\gamma y
$$

5Julio Santos-Cayado. "Ecuaciones Navier-Stokes'. Conferencias INTEC, Santo Domingo, marzo 1981. 
El que aparezca o no la componente del término de la fuerza con servativa $f$, en la ecuación 6 , depende de la orientación de los ejes coordenados.

Si $y^{\prime}=y+z$

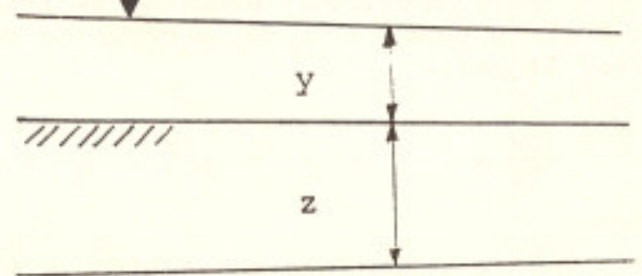

Se obtiene:

FIG. 1

$\frac{1}{\rho} \frac{\partial p}{\partial x}=\frac{\partial}{\partial x}(y+z)=g\left(\frac{\partial y}{\partial x}+\frac{\partial z}{\partial x}\right)$

Donde,

$$
\frac{\partial z}{\partial x}=-s_{0}
$$

Subst i tuyendo:

$$
\frac{\partial u}{\partial t}+u \frac{\partial u}{\partial x}=-g \frac{\partial y}{\partial x}+g S_{0}
$$

Si endo,

$$
\begin{aligned}
& S_{f}=\frac{d}{d x}\left(y+z+\frac{u^{2}}{2 g}\right) \\
& =\frac{d y}{d x}+\frac{d z}{d x}+\frac{u d u}{g d x}=\frac{\partial y}{\partial x}+\frac{\partial z}{\partial x}+\frac{u}{g}\left(\frac{\partial u}{\partial x}+\frac{\partial u}{\partial t} \frac{\partial t}{\partial x}\right) \\
& =\frac{\partial y}{\partial x}+\frac{\partial z}{\partial x}+\frac{u}{g} \frac{\partial u}{\partial x}+\frac{1}{g}-\frac{\partial u}{\partial t}
\end{aligned}
$$

Por tanto, se puede escribir:

$$
S_{f}=S_{0}-\frac{\partial y}{\partial x}-\frac{u}{g} \frac{\partial u}{\partial x}-\frac{1}{g} \frac{\partial u}{\partial t}=\frac{u^{2}}{c^{2} R}
$$


Uniforme y

permanente

Permanente, no uniforme

No permanente, no uniforme

$$
C=\text { Coeficiente de Chezy }
$$

FIG. 2

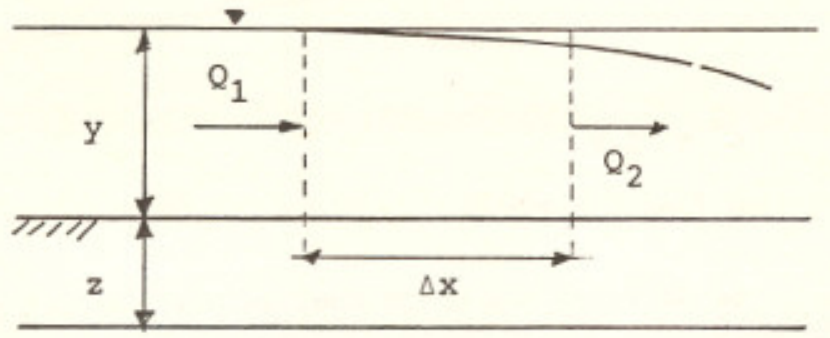

La ecuación de continuidad:

$$
\frac{\partial Q}{\partial x}+B \quad \frac{\partial y}{\partial t}=0
$$

Si $Q=B . q$, siendo $B$ el ancho del canal.

$$
\frac{\partial q}{\partial x}+\frac{\partial y}{\partial t}=0
$$

Como $q=u \cdot y$

$$
u \frac{\partial y}{\partial x}+y \frac{\partial u}{\partial x}+\frac{\partial y}{\partial t}=0
$$

Si introducimos en las ecuaciones 13 y 17 , denominadas Saint-Ve nant, la celeridad de una perturbación desplazándose en el canal con profundidad $y$. 


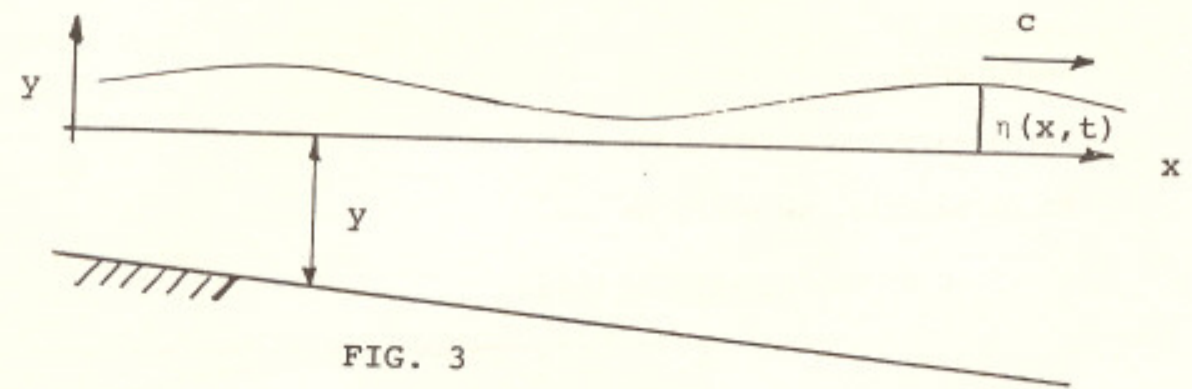

$$
\begin{aligned}
& c^{2}=g \cdot y \\
& d(g y)=2 c d c
\end{aligned}
$$

Se obtienen las ecuaciones de movimiento y continuidad:

$$
\begin{aligned}
& 2 c \frac{\partial c}{\partial x}+u \frac{\partial u}{\partial x}+\frac{\partial u}{\partial t}=g\left(s_{0}-s_{f}\right) \\
& 2 u \frac{\partial c}{\partial x}+c \frac{\partial u}{\partial x}+2 \frac{\partial c}{\partial t}=0
\end{aligned}
$$

Si se restringen las ecuaciones 20 y 21 a movimientos que difieren poco de la situación de equilibrio, $n=0$; $u=0$, se puede obtener la ecuación lineal clásica de la onda, veamos:

$$
\begin{aligned}
& c=\sqrt{g(n+y)}=\sqrt{g y}+\sqrt{g n} \\
& c=c_{0}+\varepsilon(x, t)
\end{aligned}
$$

Asumiendo $\varepsilon$, u y sus derivadas de primer orden, asumiendo as imismo, $y=$ constante, tendremos:

$$
\begin{aligned}
& \frac{\partial u}{\partial t}+2 c_{0} \frac{\partial \varepsilon}{\partial x}=0 \\
& 2 \frac{\partial \varepsilon}{\partial t}+c_{0} \frac{\partial u}{\partial x}=0
\end{aligned}
$$


Derivando con relación a $t$ y a $x$ respectivamente, eliminando $\varepsilon$, $y$, reteniendo sólo los términos de primer orden:

$$
\frac{\partial^{2} u}{\partial t^{2}}+2 c_{0} \frac{\partial^{2} \varepsilon}{\partial \times \partial t}=0
$$

de donde:

$$
\begin{gathered}
\frac{\partial^{2} \varepsilon}{\partial x \partial t}=-\frac{1}{2 c_{0}} \frac{\partial^{2} u}{\partial t^{2}} \\
2 \frac{\partial^{2} \varepsilon}{\partial t \partial x}+c_{0} \frac{\partial^{2} u}{\partial x^{2}}=0
\end{gathered}
$$

Substituyendo la 27 en la 28 y rearreglando:

$$
\frac{\partial u}{\partial t^{2}}-c_{0}^{2} \frac{\partial^{2} u}{\partial x^{2}}=0
$$

Conocida como la ecuación de D'Alembert, resuelta el siglo pasa do, cuyas soluciones son de la forma $u=u(x \pm c t t)$, lo que significa que los movimientos son superposiciones de ondas con celeridades constantes. Esto puede "verse" matemáticamente, si sumamos y restamos las ecuaciones 20 y 21 :

$$
\begin{aligned}
& \frac{\partial u}{\partial t}+(u+c) \frac{\partial u}{\partial x}+2 \frac{\partial c}{\partial t}+2(u+c) \frac{\partial c}{\partial x}=g\left(s_{o}-s_{f}\right)(30) \\
& \frac{\partial u}{\partial t}+(u-c) \frac{\partial u}{\partial x}-2 \frac{\partial c}{\partial t}-2(u-c) \frac{\partial c}{\partial x}=g\left(s_{o}-s_{f}\right)(31)
\end{aligned}
$$

En las que los miembros de la izquierda son derivadas totales, por tanto:

$$
\frac{D_{1}(u+2 c)}{D_{1} t}=g\left(s_{o}-s_{f}\right)
$$




$$
\frac{D_{2}(u-2 c)}{D_{2} t}=g\left(s_{o}-S_{f}\right)
$$

Los operadores derivadas totales $D / D$ t representan las velocidades de cambio que "ven" observadores moviéndose con velocidades (u+c) - $(u-c)$ respectivamente. Las trayectorias de estos observadores pueden ser mapeadas en el plano $x-t$, resultando en una solución completa para cualquier situación no permanente.

For razones de las condiciones iniciales y de borde, sólo en los casos más simples, el proceso conduce a una solución explícita. En los demás casos, las soluciones a estos problemas se obtienen de la combinación de procedimientos gráficos y numéricos, calibradas esas so luciones, por modelos hidráulicos y experiencias de laboratorio y/o de campo.

\section{Aplicaciones}

Para ilustrar el uso de las ecuaciones de las características, 32 y 33 , a fin de desarrollar un sentimiento primario de cómo se desenvuelve el problema de rotura de una presa, resolveremos dos casos sencillos. Un tratamiento más profundo corresponde a un curso formal; no obstante, en el acápite siguiente incluimos dos casos reales.

1. Falla una presa localizada en un canal rectangular seco, de pendiente y rugosidad negligibles.

En este caso los miembros de la derecha de las ecuaciones 32 y 33, se hacen cero. Por tanto, los observadores que se mueven con velocidad $(u+c)$, "verán" que las velocidades de cambio que representan las derivadas totales, permanecerán constantes, iguales a $(u \pm 2 c)$.

Las trayectorias que siguen esos observadores se denominan características, existiendo dos familias de ésas, la C1, determinada por observadores en las líneas rectas definidas por las pendientes $1 /(\mathrm{u}+\mathrm{c})$ y 1 las C2 que son curvas. (Ver Figura 4, p. 219).

Se trazan, en este caso, desde el origen, las características correspondientes a la familia C1. Para determinar la característica que separa la zona perturbada del embalse, de la tranquila, se nota que en ésta, $u=0$, por tanto, su pendiente será $1 / c_{0}$, siendo $c_{0}=\sqrt{g \cdot y_{0}}$.

La característica que define la puntera de la ola, se determina combinando los valores de las pendientes o sus inversos para las C1. 


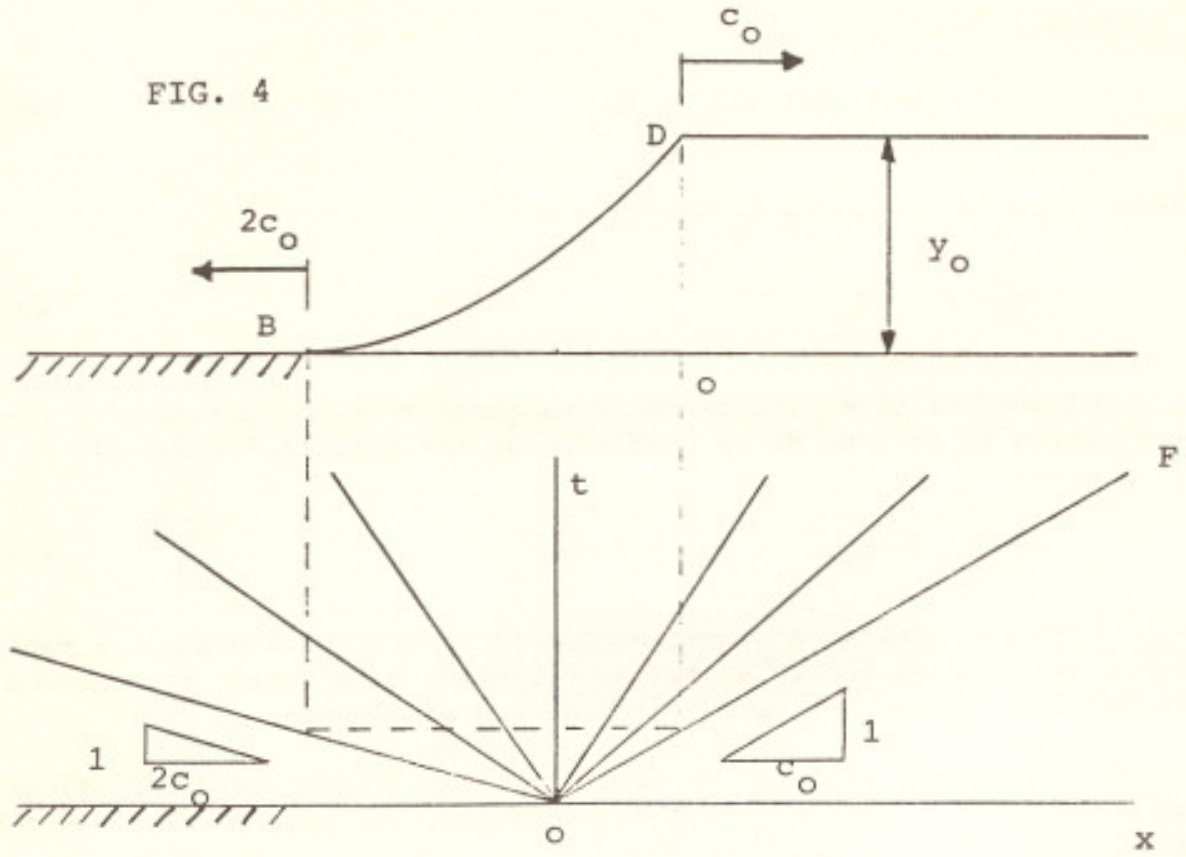

$$
\frac{d x}{d t}=u(t)+c(t)
$$

con los valores constantes de las velocidades de cambio a lo largo de las características $\mathrm{C} 2$, establecidos para un punto sobre la C1 conoci da y otro sobre la buscada:

$$
u(t)-2 c(t)=u_{0}-2 c_{0}
$$

De donde:

$$
2 c(t)=\frac{1}{2} u(t)-\frac{1}{2} u_{0}+c_{0}
$$

Por tanto:

$$
\frac{d x}{d t}=\frac{3}{2} u(t)-\frac{1}{2}+c_{0}
$$


o también:

$$
=3 u(t)+u_{0}-2 c_{0}
$$

Siendo para la divisoria $u_{0}=u(t)=0$

$$
\frac{d x}{d t}=-2 c_{0}
$$

El perfirl $B D$ es una parábola tangente al fondo del canal y cuya ecuación se obtiene de la pendiente de las características C1:

$$
\frac{x}{t}=3 \sqrt{g \cdot y}+u_{0}-2 \sqrt{g \cdot y_{0}}
$$

El flujo constante producido por la rotura de la presa se mantiene hasta que el frente de la perturbación, D, alcanza el extremo del embalse. Luego, el caudal disminuye gradualmente.

2. La presa falla en un canal con las mismas características que en el caso 1, con excepción de que ahora, hay agua en reposo en el canal.

Este caso, tal como indica su planteamiento, es esencialmente igual al interior y quedaría resuelto si definimos el efecto del agua en el canal sobre la puntera de la ola. (Ver Figura 5, P. 221).

Si la parábola CD fuera a tocar directamente la superficie del agua en el canal, se ocasionaría una discontinuidad en las velocidades en ese punto de contacto. Esta discontinuidad sólo es posible a través de un salto pronunciado en el perfil, es decir de una ola de al tura finita.

Una vez que se forma la ola, su extremo aguas abajo, alcanza ma yor velocidad que el c, por lo que la ola aumenta constantemente en long i tud.

\section{Efectos de Pendiente y Rigurosidad.}

En referencia particular al caso 1, más arriba, se entiende que los razonamientos originados por la rigurosidad del cauce, impiden que la parábola BD sea tangentę a su fondo, sino que formará un ángulo más o menos pronunciado en él.

6.M. Henderson. Open Channel Flow. New York: The Macmill lan Co. 


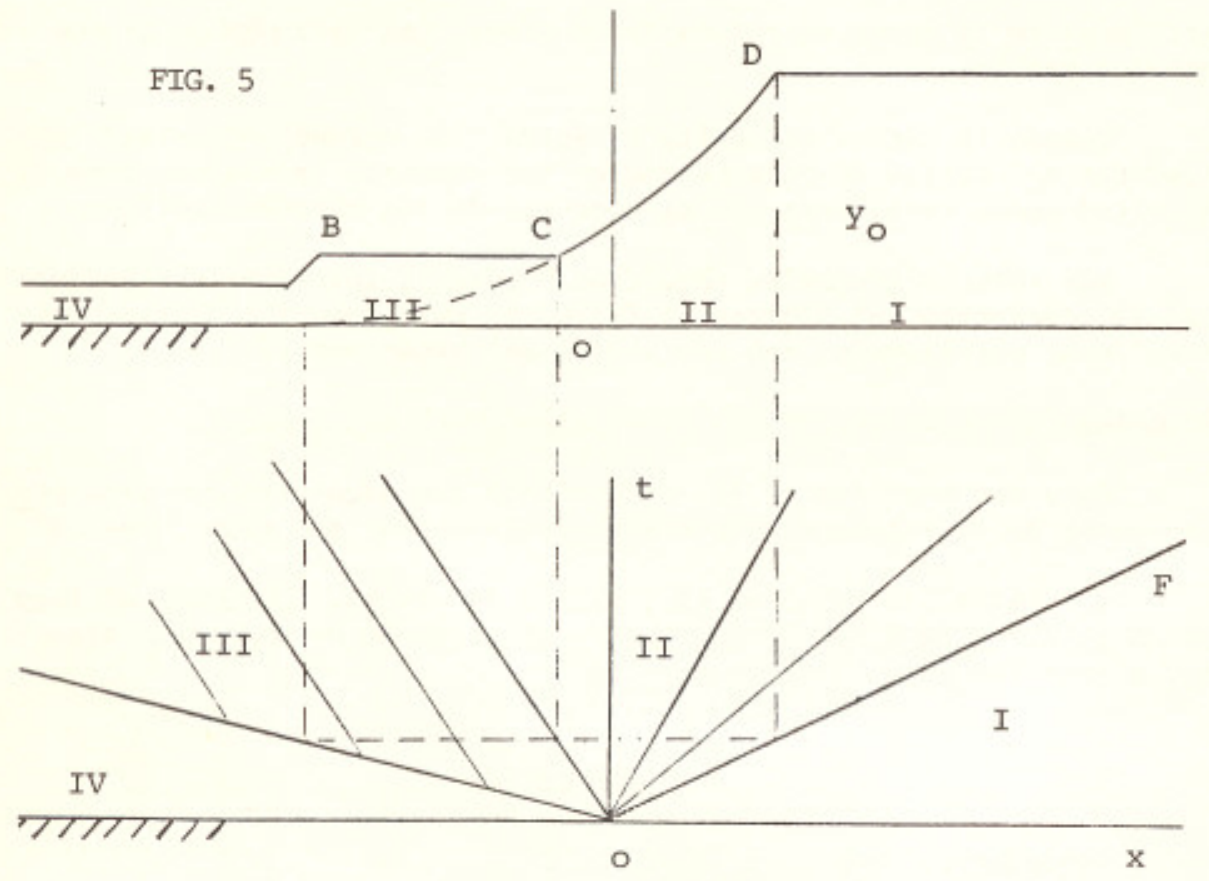

En el caso general de la solución del problema de rotura de pre sa, vemos que los miembros derechos de las ecuaciones 32 y 33 , no se
anulan.

La permanencia en las ecuaciones de $S_{o}$ y $S_{f}$ complica considerablemente el problema. En el caso de $S_{f}$ porque sus valores dependen de una manera que no conocemos de algunas de las variables que estamos tratando de determinar, $y, u$, por ejemplo.

Los valores de $\mathrm{S}_{0}$, pendiente del canal, no son uniformes, $\mathrm{ni}$ a lo largo del canal, ni en sus secciones transversales, de manera que los levantamientos topográficos han de limitarse a obtener la pendien te media, por tramos, del thalweg.

Estas dos razones básicas hacen que para la solución de casos reales, sea necesario combinar el método gráfico, con procedimientos numéricos de iteración y modelos hidráulicos.

4. Rotura Súbita o Gradual.

A los inconvenientes descritos en el punto 3 , se agregan los que 
provienen de la forma de rotura de la presa, sea ésta rápida, gradual, total o parcial.

Cuando la rotura es súbi ta o rápida, los métodos numéricos encuentran dificultad de continuidad en los momentos iniciales. Esta di ficultad puede resolverse con el concurso de los métodos gráficos.

Las roturas graduales requieren hipótesis iniciales que produzcan la modelación del proceso de fallo, en este caso los modelos hidráulicos, por ejemplo, dan una visión del mecanismo físico.

Ejemplos

Para dar una idea de las dimensiones que adquieren las olas pro venientes de la rotura de una presa, resolveremos dos casos "reales".

1. Falla una presa de 40.0 metros de altura, formando un lago de unos 10.0 kilómetros de longitud y descargando en un canal trapezoidal con las siguientes dimensiones.

FIG. 6
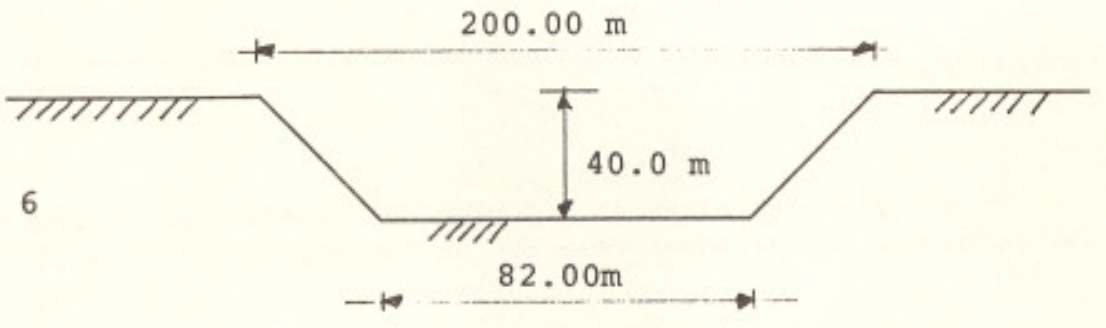

de donde:

$$
R=37.0 \text { metros }
$$

y con una pendiente de rugosidad:

$$
\begin{aligned}
& s_{f}=0.00769 \\
& n=0.030
\end{aligned}
$$

7J. G. Sakkas, T. Strellkoff. "Dimensionless Solution of DamBreak Flood Waves", Journal of the Hydraulic Division ASCE. Vol. 102, Proc. Paper 11910, febrero 1976. 
De donde:

$$
\begin{aligned}
& V_{0}=32.50 \text { metros } / \text { segundo } \\
& T_{0}=\frac{10,000}{32.50}=307.73 \text { segundos; } 5 \text { min., } 8 \text { seg. }
\end{aligned}
$$

Si la distancía a la ciudad más próxima es de $26.0 \mathrm{Km}$.

Se obtienen:

Tiempo de llegada del pie de ola : $21 \mathrm{~min} ., 44 \mathrm{seg}$.

Tiempo de llegada del pico de ola : $27 \mathrm{~min}$, , $41 \mathrm{seg}$.

Altura máxima de ola (cresta) : 14 metros.

2. Si la presa que falla es igual a la anterior, pero el cauce tiene las siguientes dimensiones:

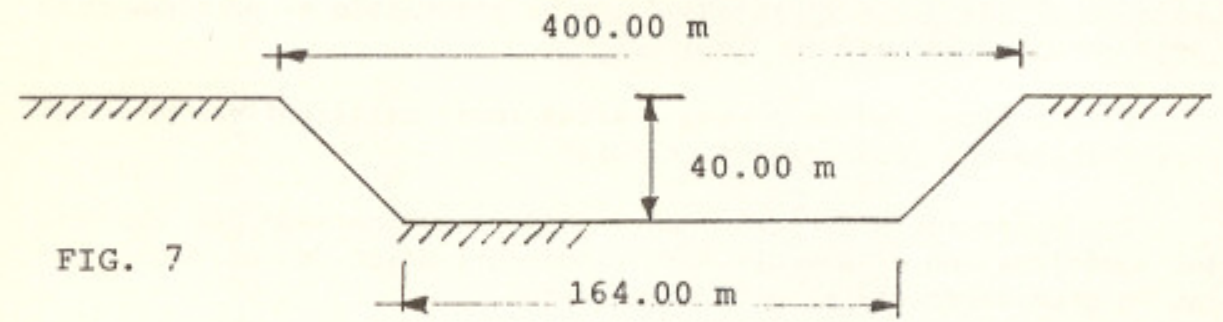

$$
\begin{aligned}
& R=27.30 \mathrm{~m} . \\
& S_{f}=00.769 \\
& n=0.040 \\
& V_{0}=19.86 \mathrm{~m} / \mathrm{s} \\
& T_{0}=366.3 \mathrm{seg}, 6 \mathrm{~min} .6 \mathrm{seg} .
\end{aligned}
$$

Tiempo de llegada del pie de ola a ciudad : $26 \mathrm{~min}$.

Tiempo de llegada de la cresta de la ola: $33 \mathrm{~min}$.

Altura máxima de ola (cresta) : 14 metros.

3. Si todo permaneciera igual, pero el cauce fuera más rugoso: 


$$
\begin{aligned}
& n=0.070 \\
& V_{0}=11.35 \mathrm{~m} / \mathrm{s} \\
& T_{0}=881.17 \mathrm{seg}, 14 \mathrm{~min} ., 41 \mathrm{seg} .
\end{aligned}
$$

Tiempo de llegada del pie de ola a la ciudad: $62 \mathrm{~min}, 25 \mathrm{seg}$. Tiempo de llegada de la cresta: $79 \mathrm{~min}, 18 \mathrm{seg}$. Altura máxima de ola (cresta)

: 14 metros.

\section{Conclusiones}

Aunque este problema fue estudiado por primera vez en 1882 por Ritter y en 1946 Ré publicó sus trabajos durante la Segunda Guerra Mundial, en los que hizo contribuciones significativas, no ha sido hasta la década del setenta cuando se ha presentado el problema en su justa perspectiva, por dos razones:

1. La necesidad de prevenir situaciones catastróficas de vidas y de propiedades cada vez más costosas.

2. Apoyo recibido por el desarrollo experimentado por los méto dos numéricos con el advenimiento y popularización de las computadoras de gran capacidad y de alta velocidad.

El pensamiento de algunos autores sugiere la incorporación de estudios de rotura de presa en el compromiso de diseño, con el objeto de elaborar planes de contingencia y, además, de cargar al costo de la presa una cuota anual que cubra el seguro contra accidentes a pagar por reparaciones causadas por los daños que podrían ocasionarse.

En la República Dominicana hay presas localizadas aguas arriba de ciudades importantes, por lo que resulta impostergable el conocimiento de lo que pasaría en ésas si ocurriera algún fallo.

\section{BIBLIOGRAFIA}

Ballofet, A., E. Cole y A. F. Ballofet. "Dam Collapse Wave in a River". Journal of the Hydraulic Division, ASCE. 100 (HY5) Proc., Paper 10523, mayo 1974. 
Fread, D., L. y T.E. Harbaugh. "Transient Hydraulic Simulation of the Breached Earth Dam." Journal of the Hydraulic Division, ASCE, (HY1) Proc., Paper 9466, enero 1976.

Henderson, F., M. Open Channel Flow. New York: The Macmillan Company, 1966.

Rajar, R. "Mathematical Simulation of the Dam-Break Flow," Journal of the Hydraulic Division, ASCE, 104. (HY7) Proc. Paper 13883, julio 1978.

Sakkas, J. G. y T. Strelkoff. "Dam-Break Flood in a Prismatic Dry Channel." Journal of the Hydraulic Division, ASCE, 99. (HY12) Proc. Paper 10233, diciembre 1973.

"Dimensionless Solution of Dam-Break Flood Waves." Journal of the Hy draulic Division, ASCE, 102, Proc., Paper 11910, febrero 1976.

Santos-Cayado, J., "Ecuaciones Navier-Stokes." Santo Domingo: Conferencias INTEC, marzo 1981.

Strelkoff, T., "Numerical Solution of the Saint-Venant Equations." Jowrnal of the Hydraulic Division, ASCE, 96. (HY1) Proc., Paper 70043 , enero 1970.

USCOLD/ASCE, Lessons from Dam Incidents, USA. New York: ASCE, 1975. 